\title{
Deployment of Mobile Air Sensing Network for Urban Air Pollution Monitoring in Hong Kong ${ }^{+}$
}

\author{
Fenhuan Yang ${ }^{1}$, Junke Zhang ${ }^{1}$, Yang Xing ${ }^{1}$, Jieqing He ${ }^{1}$, Kai Zhang ${ }^{1}$, Dane Westerdahl ${ }^{1}$ and \\ Zhi Ning ${ }^{2}$ * \\ 1 School of Energy and Environment, City University of Hong Kong, Hong Kong Special Administrative \\ Region; fhyang2012@gmail.com (F.Y.); junkezhang16@gmail.com (J.Z.); xy11nju@163.com (Y.X.); \\ hejieqing0815@hotmail.com (J.H.); kiwizhang1993@gmail.com (K.Z.); danewest03@gmail.com (D.W.) \\ 2 Guy Carpenter Climate Change Centre, City University of Hong Kong, Tat Chee Avenue, Kowloon, Hong \\ Kong Special Administrative Region \\ * Correspondence: zhining@cityu.edu.hk \\ + Presented at the 5th International Symposium on Sensor Science (I3S 2017), Barcelona, Spain, \\ 27-29 September 2017.
}

Published: 14 December 2017

Epidemiology studies have demonstrated an association between airborne pollutant and adverse effects on human health. People living and working in metropolitan cities are directly exposed to air pollution from surrounding environment. In the meantime, vehicle emissions contribute a larger portion to urban air pollution. The increased vehicle use gives rise to an increase in traffic related pollutant emission such as $\mathrm{CO} / \mathrm{CO}_{2}, \mathrm{NOx}$ and particulate matters (PM).The present study employed a mobile air sensing network (MASEN) with high resolution and precision in monitoring on-road pollution in Hong Kong. It could be a complementary method of common pollutant measurement in terms of updating pollutant distribution map at specific time interval. A cross comparison was also carried out with regulatory stationary monitoring stations. The result showed gas pollutant was moderate correlated with traffic volume $(0.4<\mathrm{r}<0.6, p<0.05)$ but PM distributed more evenly citywide. The result also found that on-road pollutant was consisted of local background pollutant and foreign transported pollutant. Local background pollutant was correlated with urban monitoring station background while foreign transported pollutant was dominated by on-road environment. Pollutant concentration hot spots were also identified and analyzed in the result. The present study provides a better understanding of on-road pollutant distribution as well as its major affection factors. The low emission zones (LMZ) implementation and traffic emission control policy are discussed with recommendations for future measurement and policy making.

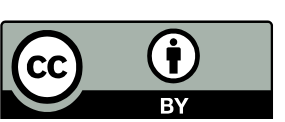

(C) 2017 by the authors. Licensee MDPI, Basel, Switzerland. This article is an open access article distributed under the terms and conditions of the Creative Commons Attribution (CC BY) license (http://creativecommons.org/licenses/by/4.0/). 